\title{
Evaluación de la formación mediante indicadores de calidad
}

\section{Quality indicators for evaluation training}

\author{
Jesús Miguel Muñoz-Cantero, Eva María Espiñeira-Bellón, Nuria Rebollo-Quintela \\ Departamento de Filosofía y Métodos de Investigación en Educación, Universidade da Coruña, España
}

\begin{abstract}
Resumen
En los actuales títulos, el profesorado que dirige el Trabajo Fin de Grado/Máster (TFG/TFM), ha evidenciado lagunas formativas en conocimientos específicos y competencias relativas a expresión y comunicación. El Grupo de Investigación y Evaluación de la Calidad Educativa (GIACE) ha desarrollado una actividad formativa innovadora denominada "Píldoras formativas para la realización del TFG/TFM” con varias píldoras. Considerando la formación un servicio, se ha elaborado una escala Likert que evalúa varios factores de satisfacción. Los resultados permiten establecer un modelo de evaluación de la formación mediante un sistema de indicadores que representan factores de satisfacción. Palabras clave: indicadores, calidad, evaluación, formación, educación superior.
\end{abstract}

\section{Abstract}

In the current titles, teachers who heads the Final Degree/Master

(TFG/TFM) work has demonstrated specific training gaps in knowledge and skills related to expression and communication. The Group of Research and Evaluation of Educational Quality (GIACE) has developed an innovative training activity called "Training pills for carrying out the TFG/TFM" with various pills. Considering training services, it has developed a Likert scale that assesses several factors of satisfaction. The results indicate a model of evaluation of training through a system of indicators that represent factors of satisfaction.

Keywords: indicators, quality, evaluation, training, higher education.

El Espacio Europeo de Educación Superior (EEES) y el diseño de los títulos, de acuerdo a la normativa específica de cada país, exige que, al finalizar los estudios de grado o de máster, el estudiantado tenga que realizar un Trabajo de Fin de Grado (TFG) o Máster (TFM) para obtener su título. Así, en España, el Real Decreto 43/2015, de 2 de febrero, por el que se modifica el Real Decreto 1393/2007, de 29 de octubre, por el que se establece la ordenación de las enseñanzas universitarias oficiales, y el Real Decreto 99/2011, de 28 de enero, por el que se regulan las enseñanzas oficiales de doctorado, recoge que dichas enseñanzas concluirán con la elaboración y defensa de un TFG/TFM.
Varios años después de la implantación de estos títulos en la Facultad de Ciencias de la Educación de la Universidad de A Coruña, el profesorado ha detectado individualmente, en las tutorías de dirección de dichos trabajos, diversas necesidades formativas. Para paliarlas, el Grupo de Investigación y Evaluación de la Calidad Educativa (GIACE), del Departamento de Filosofía y Métodos de Investigación en Educación de dicha Universidad, ha desarrollado una actividad formativa, de carácter innovador, que ha denominado "Píldoras formativas para la realización del TFG/TFM”. Dicha actividad se compone de varias píldoras.

Las píldoras de conocimiento, como indican Romero-Rameta, Girela-López, Gómez Torres, Segovia Huertas, Martínez Ruiz y de Juan Herrero (2012), consisten en cursos breves que sirven de orientación sobre temas específicos y se expresan, por lo tanto, como unidades independientes para entender un concepto. En la presente actividad formativa, se han desarrollado como “mini píldoras formativas” (Aranguena, Ruiz y Urrutia, 2010, p. 12) a través de sesiones cortas (de hora y media de duración) intentando interrumpir lo mínimo el ritmo de trabajo del alumnado durante la jornada (se han celebrado por las tardes, cuando el alumnado no tenía que acudir a sesiones de clase). La formación se ha acompañado asimismo de una presentación en powerpoint sencilla para el apoyo a la explicación, a la cual el alumnado puede acudir a través de la página web del Grupo cada vez que lo necesite.

No obstante, como indican Bravo (1994) y Dorado (2006), han de emplearse en un contexto en el que produzcan aprendizaje, formando parte de un determinado módulo formativo. En este sentido, Borrás Gené (2012) las define como "la unidad más pequeña de información dotada de significado y totalmente independiente, de tal manera que se pueda utilizar de forma autónoma. A su vez, también puede formar parte de un curso más extenso” (p. 15). De esta forma, las píldoras formativas diseñadas se integran en una actividad formativa más amplia dedicada a la realización de los TFG/TFM.

Por otra parte, dado que la formación puede considerarse un servicio, debe estar sujeta a un mecanismo de evaluación válido y fiable tal como se recoge desde cualquier sistema de calidad. Como señala Hilera González (2008), ya han empezado a desarrollarse estándares internacionales relacionados con la calidad en la formación en general, como la norma ISO 19796-1:2005. 
Con el fin de mejorar la satisfacción de los/las estudiantes que han asistido a la actividad formativa, en el presente trabajo se establece un estándar. El nivel de satisfacción dependerá de la diferencia positiva o negativa, entre sus expectativas iniciales (lo que esperaban) y lo que han recibido. El modelo de calidad establecido por este estándar se basa en el ciclo de satisfacción de las necesidades y expectativas de clientes de AENOR (2008). (Véase Figura 1).

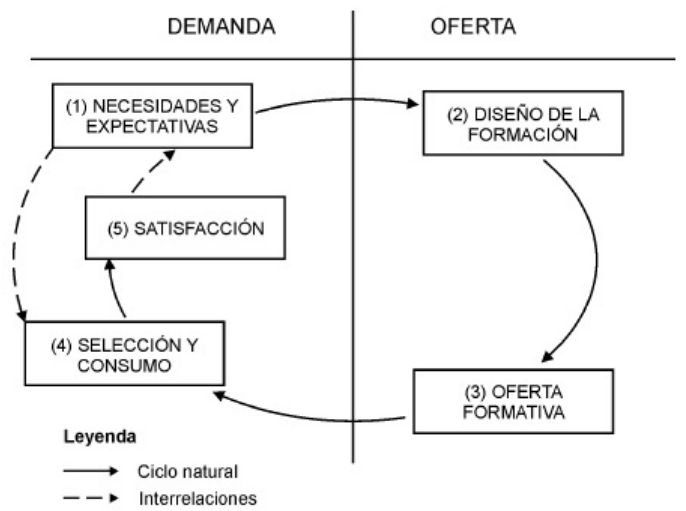

Figura 1. Ciclo de satisfacción de necesidades y expectativas de los clientes de la formación virtual

De acuerdo a la figura anterior, el ciclo comienza cuando las necesidades formativas son detectadas, analizadas y utilizadas como guía para el diseño y desarrollo de la oferta formativa demandada. La formación desarrollada será ofertada, de forma que pueda ser analizada por las personas usuarias. La satisfacción de sus necesidades y expectativas estará directamente relacionada con su capacidad para seleccionar la oferta formativa más adecuada a su situación. Las personas usuarias seleccionarán la oferta formativa más interesante para ellas y, una vez recibida la formación, aumentará o disminuirá su satisfacción en función de la diferencia positiva o negativa entre sus expectativas y lo recibido.

El anterior modelo ha sido tomado como referencia para diseñar el proceso de evaluación de las píldoras formativas puestas en marcha. Se desarrollan a continuación el método empleado, el instrumento diseñado, procedimiento, resultados, conclusiones y discusión.

\section{Método}

En el curso 2014/2015, el Grupo GIACE detecta diversas necesidades formativas en las tutorías de los TFG/TFM que dirige, las cuales se centran en la falta de conocimientos específicos y en otros tipos de competencias relativas a la expresión y a la comunicación.

Una vez detectadas y analizadas dichas necesidades diseña una oferta formativa denominada "Píldoras formativas para la realización del TFG/TFM”; que, como su nombre indica, se compone de varias píldoras: 1) el TFG/TFM y el proceso de investigación, 2) abordar una investigación cualitativa, 3) organiza los datos de tu cuestionario, 4) analiza tus datos con estadística básica,
5) representa tus datos mediante gráficos/tablas, 6) aprende a almacenar, recuperar y compartir recursos, 7) mejora tu estilo de redacción y aprende a citar, 8) cómo hacer la presentación del TFG/TFM, 9) tono y voz en la presentación de tu trabajo.

La formación ha sido ofertada de manera general a todo el alumnado que curse la materia de TFG/TFM en cualquiera de los Grados y Masters de la Facultad de Ciencias de la Educación de la Universidad de A Coruña. Para ello, se ha recurrido a mails informativos y al diseño de varios posters ubicados en diferentes dependencias de la Facultad. La información ha podido ser analizada por el alumnado interesado que ha seleccionado aquellas píldoras más adecuadas a sus necesidades y situación particular.

Finalmente, se recoge la información acerca de la satisfacción del alumnado mediante el instrumento diseñado que se desarrollará posteriormente.

\section{Participantes}

Las personas participantes en la presente evaluación de la acción formativa han sido 41 (100\% de mujeres) asistentes a las píldoras formativas, de diferentes titulaciones que se imparten en la Facultad de Ciencias de la Educación de la Universidad de A Coruña, mayoritariamente del Grado en Educación Infantil. (Véase Figura 2).

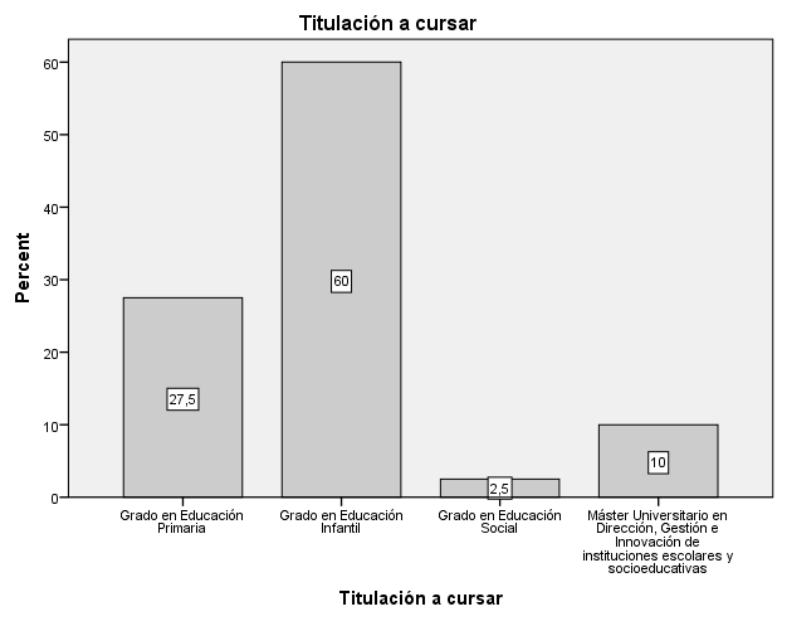

Figura 2. Titulación cursada

La selección de la muestra se estableció mediante muestreo no probabilístico de carácter incidental ya que se aplicó a todas las alumnas que asistieron a recoger el certificado acreditativo de haber realizado las píldoras formativas.

\section{Instrumento}

Partiendo del ciclo de satisfacción de necesidades y expectativas (Véase Figura 2), se desarrolla un modelo basado en una serie de indicadores de calidad que representan factores de satisfacción de éstos, cada uno de los cuales se descompone en atributos clave sobre los que se puede actuar para mejorar el factor de satisfacción correspondiente, tal como se muestra en la Tabla 1. 
Tabla 1.

Factores de satisfacción y atributos clave

\begin{tabular}{lc}
\hline Factores de satisfacción & Atributos clave \\
\hline Información & Información básica \\
& $\begin{array}{c}\text { Reconocimiento de la } \\
\text { información } \\
\text { Demanda de la información } \\
\text { Transferencia de los } \\
\text { conocimientos } \\
\text { Aplicabilidad }\end{array}$ \\
& Valor añadido \\
& Competencia académica \\
Facilidad de asimilación & Estimulación al aprendizaje \\
& A los contenidos \\
Accesibilidad & Al profesorado \\
Capacidad de respuesta & A los/las responsables de la \\
Fiabilidad & Vestión de la acción formativa \\
Competencia técnica & Acciones desarrolladas \\
Empatía & Conocimientos profesorado \\
Credibilidad & Cercanía \\
Impacto & Confianza profesorado \\
\hline
\end{tabular}

Para cuantificar en qué grado se satisfacen las necesidades y expectativas del alumnado, se han establecido diez factores de satisfacción críticos: información (establece un conjunto de datos que han de suministrarse al estudiantado ante la oferta de la acción formativa -trípticos y posters-; aplicabilidad (valor añadido, competencia académica) representa en qué medida la formación incrementa la capacidad del alumnado de resolver satisfactoriamente el TFG/TFM o aumenta su competencia técnica para resolver problemas; facilidad de asimilación (capacidad de la acción formativa para estimular al usuario con el fin de entender los contenidos y favorecer el aprendizaje); accesibilidad (facilidad del estudiantado para mantener contacto con el profesorado y responsables de la acción formativa); capacidad de respuesta (velocidad de respuesta ante las solicitudes de los/las estudiantes); fiabilidad (capacidad de realizar la acción formativa comprometida); competencia técnica (conocimientos del profesorado para realizar la acción formativa); empatía (cercanía demostrada por el profesorado y responsables de las acciones formativas); credibilidad (confianza que trasmite el profesorado respecto a los contenidos trasmitidos); impacto (previsión de que la formación tendrá efectos positivos sobre los resultados obtenidos en la realización y defensa del TFG/TFM).

Como punto de referencia, se ha tomado la literatura sobre calidad de servicio percibida y los estándares internacionales relacionados con la calidad en la formación en general (AENOR, 2005) y en particular sobre formación en entornos virtuales de aprendizaje debido a que las píldoras formativas están siendo utilizadas mayoritariamente en estos entornos (AENOR, 2008; Hilera González, 2008; Martínez-Argüelles, Blanco Callejo, y Castán Farrero, 2013).
En el estándar diseñado, los factores de satisfacción se presentan en una graduación de cuatro niveles de calidad. La escala va desde el nivel 1 (nada) al nivel 4 (mucho). De esta forma, el futuro alumnado podrá obtener una valoración global del nivel de satisfacción que le puede proporcionar la oferta formativa $\mathrm{y}$, por lo tanto, seleccionar la que más se adecúa a sus necesidades y expectativas.

A mayores, se han incluido dos ítems; uno de satisfacción general, de tal manera que el nivel de calidad de cada píldora vendrá determinado por la combinación del nivel de satisfacción asociado a cada factor; otro con respecto a la decisión del alumnado de recomendar sucesivas actividades formativas.

Además se incluye una pregunta de carácter abierto con el fin de recoger información sobre necesidades futuras.

\section{Procedimiento}

El procedimiento de recogida de datos se ha realizado una vez finalizadas las píldoras formativas, en el momento en el cual el alumnado acude a recoger el certificado de participación en dicha acción formativa.

\section{Resultados}

El análisis de resultados se ha establecido mediante el programa de análisis estadístico IBM SPSS Statistics Versión 20.

Los análisis planteados se basan en estadística descriptiva mediante medidas de tendencia central y dispersión (media y desviación típica), en primer lugar sobre cada una de las píldoras formativas. (Véase Tabla 2).

Tabla 2.

Estadísticos descriptivos

\begin{tabular}{lccc}
\hline Píldoras formativas & $\mathrm{N}$ & $\begin{array}{c}\text { Satisfacción } \\
\text { general } \\
\text { (Media) }\end{array}$ & $\begin{array}{c}\text { Desv. } \\
\text { típica }\end{array}$ \\
\hline $\begin{array}{l}\text { El TFG/TFM y el proceso de } \\
\text { investigación }\end{array}$ & 25 & 3.28 & .678 \\
$\begin{array}{l}\text { Abordar una investigación } \\
\text { cualitativa }\end{array}$ & 23 & 3.04 & .825 \\
$\begin{array}{l}\text { Organiza los datos de tu cuestionario } \\
\text { Analiza tus datos con estadística }\end{array}$ & 19 & 3.53 & .697 \\
$\begin{array}{l}\text { básica } \\
\text { Representa tus datos mediante } \\
\text { gráficos/tablas }\end{array}$ & 17 & 3.29 & .849 \\
$\begin{array}{l}\text { Aprende a almacenar, recuperar y } \\
\text { compartir recursos }\end{array}$ & 12 & 3.17 & .937 \\
$\begin{array}{l}\text { Mejora tu estilo de redacción y } \\
\text { aprende a citar }\end{array}$ & 34 & 3.77 & .426 \\
$\begin{array}{l}\text { Cómo hacer la presentación del } \\
\text { TFG/TFM }\end{array}$ & 33 & 3.79 & .410 \\
$\begin{array}{l}\text { Tono y voz en la presentación de tu } \\
\text { trabajo }\end{array}$ & 29 & 3.66 & .484 \\
\hline
\end{tabular}


Como podemos apreciar en la tabla 2, nos encontramos con respuestas bastantes homogéneas ya que la desviación típica que presentan las diferentes píldoras, no es superior a 1 en ningún caso. No obstante, es necesario matizar que no todo el alumnado ha acudido al mismo número de píldoras formativas.

Las puntuaciones medias de los ítems comprenden unas valoraciones entre $\overline{\mathbf{x}}=3.04$ de la píldora formativa "Abordar una investigación cualitativa” y $\overline{\mathbf{x}}=3.79$ de “Cómo hacer la presentación del TFG/TFM”. Por lo que extraemos que la muestra encuestada, está bastante satisfecha con todas las píldoras formativas. Además, cuatro de ellas presentan $\overline{\mathbf{x}}>3.50$, medias muy cercanas a la satisfacción total.

En segundo lugar, los resultados permiten además establecer un modelo de evaluación de la formación a través de un sistema de indicadores que representan factores de satisfacción. (Véase Tabla 3).

Tabla 3.

Estadísticos descriptivos

\begin{tabular}{lcc}
\hline Factores de satisfacción & Media & $\begin{array}{c}\text { Desviación } \\
\text { típica }\end{array}$ \\
\hline Información & 3.39 & .621 \\
Aplicabilidad & 3.22 & .766 \\
Facilidad de asimilación & 3.21 & .768 \\
Accesibilidad & 3.45 & .646 \\
Capacidad de respuesta & 3.54 & .650 \\
Fiabilidad & 3.56 & .567 \\
Competencia técnica & 3.77 & .421 \\
Empatía & 3.50 & .621 \\
Credibilidad & 3.73 & .499 \\
Impacto & 3.28 & .801 \\
\hline
\end{tabular}

Como podemos ver en la tabla 3 , nos encontramos nuevamente con respuestas bastantes homogéneas ya que la desviación típica que presentan los ítems referentes a los factores de satisfacción, no vuelve a superar el valor 1 en ningún caso. No obstante, volvemos a matizar que no todo el alumnado ha acudido al mismo número de píldoras formativas.

Las puntuaciones medias de los ítems comprenden unas valoraciones entre $\overline{\mathbf{x}}=3.21$ del factor de satisfacción referido a "Facilidad de asimilación (capacidad de la acción formativa para estimular al usuario con el fin de entender los contenidos y favorecer el aprendizaje)" y $\overline{\mathbf{x}}=3.77$ de "Competencia técnica (conocimientos del profesorado para realizar la acción formativa)". Por lo que extraemos que la muestra encuestada, valora como bastante su satisfacción con respecto a cada uno de los factores. Además, cinco de los factores de satisfacción presentan medias muy cercanas a la satisfacción total $(\overline{\mathbf{x}}>3.50)$.

Estos resultados se refuerzan si tenemos en cuenta la valoración que realizan en cuanto a recomendaciones futuras $(\overline{\mathbf{x}}=3.57$ y desviación típica= .632).

\section{Discusión}

Como anticipábamos, la puesta en práctica del modelo permitirá mejorar la oferta formativa y con ello la satisfacción del alumnado.

El análisis de los resultados, nos ha permitido detectar los altos de niveles de satisfacción por parte de las alumnas encuestadas tanto con respecto a cada una de las píldoras formativas como con respecto a los indicadores de satisfacción.

No obstante, somos conscientes también de que deberíamos priorizar aquellas píldoras y factores de satisfacción que han obtenido medias más bajas en el orden que se establece a continuación.

Atendiendo a los resultados anteriores, el orden de priorización de mejora con respecto a las píldoras formativas, sería el siguiente: a) Abordar una investigación cualitativa, b) Aprende a almacenar, recuperar y compartir recursos, c) El TFG/TFM y el proceso de investigación, d) Analiza tus datos con estadística básica y e) Representa tus datos mediante gráficos/tablas. Será necesario además complementar esta priorización con las respuestas que las alumnas nos han facilitado a la pregunta de carácter abierto "Indica qué otras píldoras formativas propondrías para próximas ediciones” ya que de ellas se desprende que es necesario invertir más horas en las píldoras anteriormente mencionadas, atendiendo al porcentaje de respuestas siguiente: a (9.37\%), c (3.12\%), d (28.12\%), e (3.12\%) además de la referente a Mejora tu estilo de redacción y aprende a citar (3.12\%).

En cuanto a los factores de satisfacción, el orden de priorización de mejora sería el siguiente: a) Facilidad de asimilación, b) Aplicabilidad, c) Impacto, d) Información y e) Accesibilidad. Complementaremos esta priorización con las respuestas que las alumnas nos han facilitado a la pregunta de carácter abierto anteriormente mencionada, las cuales se centran en la mejora del factor b (6.25\%), señalando que las píldoras formativas deberían ser más prácticas y menos teóricas, y a la mejora del factor referente a Capacidad de respuesta, indicando que deberían empezar a impartirse con anterioridad.

Contamos, finalmente, con información que nos permitirá diseñar nuevas píldoras formativas a las que estas alumnas hacen referencia: "Aprender a gestionar el tiempo" (9.37\%), "Mejorar la capacidad de síntesis de la información” (6,25\%) y, en un 3,12\% de los casos las restantes: "Cómo redactar”, “Trabajos por proyectos”, “Métodos de categorización”, “Análisis de entrevistas”, "Unidades didácticas”, "Fundamentaciones teóricas” y “Comunicación”.

Como limitaciones y prospectiva del trabajo, nos centraremos en conseguir una mayor adecuación de la muestra (no se han conseguido por el momento el 100\% de los resultados) lo cual permitiría establecer un análisis factorial exploratorio para comprobar la consistencia interna del instrumento, la validez de éste y su capacidad explicativa. 


\section{Referencias}

AENOR (2008). UNE 66181:2008, Gestión de la Calidad. Calidad de la Formación Virtual. Madrid: Asociación Española de Normalización y Certificación.

Aranguena, E., Ruíz, P. y Urrutia, I. (2010). Retos y respuestas de la gestión de la información en la Corporación Tecnológica Tecnalia. Recuperado de http://eprints.rclis.org/15891/

Borrás Gené, O. (2012). Píldoras formativas y videojuegos aplicados al estudio de la Ingeniería Acústica. Trabajo de Fin de Máster, Universidad Politécnica de Madrid, Madrid, España. Recuperado de http://oa.upm.es/11711/

Bravo, J.L. (1994). Rendimiento de los vídeos de alta potencialidad expresiva. Comunicación y Pedagogía. 122, 23-26. Recuperado de http://www.ice.upm.es/wps/jlbr/Documentacion/Po tencia_Vid.pdf

Dorado, C. (2006). El trabajo en red como fuente de aprendizaje: posibilidades y límites para la creación de conocimiento. Una visión crítica. Educar. 37, 11-24. Recuperado de http://ddd.uab.cat/pub/educar/0211819Xn37/02118 19Xn37p11.pdf
Hilera González, J.R. (2008). UNE 66181:2008, el primer estándar sobre calidad de la formación virtual. Revista de Educación a Distancia, RED. VII, 1-6. Recuperado de http://www.redalyc.org/articulo.oa?id=547180240 03

ISO (2005). ISO/IEC 19796-1:2005, Information technology. Learning, education and training, Quality management, assurance and metrics, Part 1: General Approach. Ginebra: International Standard Organization.

Martínez-Argüelles, M.J., Blanco Callejo, M. y Castán Farrero, J.M. (2013). Las dimensiones de la calidad del servicio percibida en entornos virtuales de formación superior. Revista de Universidad y Sociedad del Conocimiento. 10 , 89-106. http://dx.doi.org/10.7238/rusc.v10i1.1411

Romero-Rameta, A., Girela-López, J.L., Gómez Torres, M.J., Segovia Huertas, Y., Martínez Ruiz, N. y de Juan Herrero, J. (2012). Vídeos cortos y aprendizaje del color de la piel a nivel celular. Actas de las Jornadas de Redes de Investigación en Docencia Universitaria (pp. 1-9). Alicante: Universidad de Alicante. http://web.ua.es/es/ice/jornadas-redes-2012/ documentos/posters/245686.pdf 